Revista Internacional de Ciencias Podológicas

ISSN: 1989-5151

http://dx.doi.org/10.5209/RICP.59824

\title{
La ecografía como valor diagnóstico y de seguimiento ante en un caso clínico de bursitis adventicia
}

\author{
González Fernández, María Luz'; Morales Lozano, María del Rosario²; Ferrer Hernández, Yosune³
}

Fecha de recepción: 12 de julio de 2017 / Fecha de aceptación: 27 de diciembre de 2017

\section{Resumen.}

Introducción: Las bursas adventicias son estructuras que se forman como mecanismo de protección frente a fuerzas físicas sobre el tejido. La reacción al estrés por una carga axial sobre los sesamoideos producirá microtraumatismos repetitivos, siendo la causa principal una excesiva flexión plantar de M1. Clínicamente observamos signos de inflamación y una masa fluctuante con dolor a la palpación.

Caso clínico: Mujer de 47 años que presenta dolor y tumefacción en la región plantar de la $1^{\mathrm{a}}$ articulación metatarsofalángica del pie derecho, de 4 semanas de evolución. En la exploración se observa aumento de volumen de la $1^{\circ}$ MTF plantar, con signos de calor, rubor, eritema con dolor a la palpación directa y a la movilidad de la articulación, que en marcha y bipedestación la hace cojear.

Se confirma ecográficamente y radiográficamente "Bursitis adventicia inflamatoria activa de etiología biomecánica"

Discusión: El tratamiento fue conservador como describen diversos autores, con resultados excelentes, utilizando soportes plantares y modificaciones en el calzado. No se administraron antinflamatorios orales ni infiltraciones locales. Conclusión: Gracias al uso de la ecografía, se ha logrado proporcionar un diagnóstico preciso con bajo coste, inocuo, en la consulta de podología, pudiendo implementar de forma temprana el tratamiento.

Palabras clave: Bursitis adventicia; Bursitis; Ecografia; Sesamoiditis; Enfermedad de Renander

\section{[en] The ultrasound as a diagnostic and following value in a clinical case of bursitis adventitious}

\footnotetext{
Abstract.

Introduction: The adventitious bursas are structures that appear in the adulthood, as a mechanism of protection against physical forces on the tissue. The reaction to stress by an axial load on the sesamoids will produce repetitive microtrauma, being the main cause an excessive plantar flexion of M1.Clinically we observed signs of inflammation and a fluctuating mass with pain on palpation.

Clinical case: We present a 47-year-old woman presented with pain and swelling in the plantar region of the 1st metatarsophalangeal joint of the right foot, of 4 weeks of evolution.

In the exploration an increase in the volume of the 1st plantar MTF was observed, with signs of heat, flushing, erythema with pain on direct palpation and joint mobility, which in running and standing caused it to limp.

It is confirmed sonographically and radiographically "Active inflammatory bowel bursitis of biomechanical etiology". Discussion: The treatment was conservative as described by various authors, with excellent results, using foot supports and shoe modifications. No oral anti-inflammatory drugs or local infiltrations were administered.

Conclusions: With the ultrasound, it has been possible to provide an accurate diagnosis with low cost, innocuous, in the chiropody consultation, being able to implement the treatment early.

Keywords: Adventitious bursitis. Bursitis. Ultrasound. Sesamoiditis. Renander's disease

Profesora Titular de Escuela Universitaria. Facultad de Enfermería Fisioterapia y Podología. Universidad Complutense de Madrid (coautor). Dirección: Facultad de Enfermería, Fisioterapia y Podología. $3^{a}$ planta. Facultad de Medicina. Universidad Complutense de Madrid. 28040. Madrid

Luzalez@ucm.es

2 Profesora Titular de Escuela Universitaria. Facultad de Enfermería Fisioterapia y Podología. Universidad Complutense de Madrid (coautor). Dirección: Facultad de Enfermería, Fisioterapia y Podología. $3^{\text {a }}$ planta. Facultad de Medicina. Universidad Complutense de Madrid. 28040. Madrid rmorales@ucm.es

3 Colaboradora en la búsqueda bibliográfica. Alumna $4^{\circ}$ curso. Universidad Complutense de Madrid.
} 
Los autores declaran no tener ningún tipo de interés económico o comercial.

Sumario: 1. Introducción. 2. Caso clínico. 3. Discusión. 4 Conclusión. 5. Bibliografía.

Cómo citar: González Fernández, M. L.; Morales Lozano, M. R.; Ferrer Hernández, Y. (2018) La ecografía como valor diagnóstico y de seguimiento ante en un caso clínico de bursitis adventicia, en Revista internacional de ciencias podológicas $12(2), 55-62$

\section{Introducción}

Las bursas adventicias, son estructuras que aparecen en la edad adulta, como mecanismo de protección frente a la presión, fricción y cizallamiento del tejido conectivo fibroso superficial sobre las superficies óseas subcutáneas ${ }^{1-4}$.

Aunque es más frecuente en el sexo femenino no se encuentra influencia de edad ${ }^{4}$.

Existen diferentes etapas en la formación de las bursas adventicias, al inicio se produce una pequeña bursa sin cavidad, formada por vasos sanguíneos y células inflamatorias, que dará lugar a una pequeña cavidad; posteriormente esta cavidad irá adquiriendo un mayor tamaño, hasta llegar a ser independiente ${ }^{2-3}$. Estas bursas están revestidas por células columnares ${ }^{1}$ pero, carecen de un verdadero revestimiento sinovial endotelial; en su interior se produce un proceso de degeneración mucoide y mixomatosa del tejido conectivo que es la característica de la histología de las bursas adventicas ${ }^{3}$.

En la exploración clínica se observa una zona o región, correspondiente a la bursa inflamada, con tumor, en ocasiones rubor, observándose una masa fluctuante asociada a dolor localizado a la palpación y presión ${ }^{3}$

La localización más frecuente es en la cara medial de la primera cabeza metatarsal y plantar a las cabezas metatarsales, así como, en sesamoideos ${ }^{1}$, aunque pueden desarrollarse casi en cualquier parte del pie ${ }^{2}$.
La reacción al estrés por una carga axial, sobre los sesamoideos va a producir microtraumatismos repetitivos ${ }^{5}$ estos se pueden producir por múltiples acusas, entre ellas la existencia de una excesiva flexión plantar de la primera cabeza metatarsal M1, una parábola metatarsal inadecuada, una morfología estructural de un pie cavo, asociado a la plantarflexión anormal del primer radio y, el uso zapatos de tacón alto ${ }^{5-7}$. Esta situación puede provocar entre otras consecuencias: la inflamación capsular, inflamación con aparición de una bursa adventicia y el engrosamiento de la cara plantar del sesamoideo ${ }^{5,6}$.

\section{Caso clínico}

Mujer de 47 años que acude a la consulta de la Unidad Pie Reumático de la UCM Clínica Universitaria de Podología. Presentando dolor $\mathrm{y}$ tumefacción en la región plantar de la primera articulación metatarso-falángica del pie derecho de 2 semanas de evolución (Figura1)

Valorada en urgencias hospitalaria, tratada con vendaje y Aines, sin mejoría. Entre los antecedentes la paciente refiere episodios repetidos de dolor plantar en la articulación metatarso-falángica del hallux de un año de evolución. El dolor aumenta con la marcha prolongada y no refiere antecedentes traumáticos. Escala de dolor EVA $7^{8}$ en reposo y 9 en marcha. 

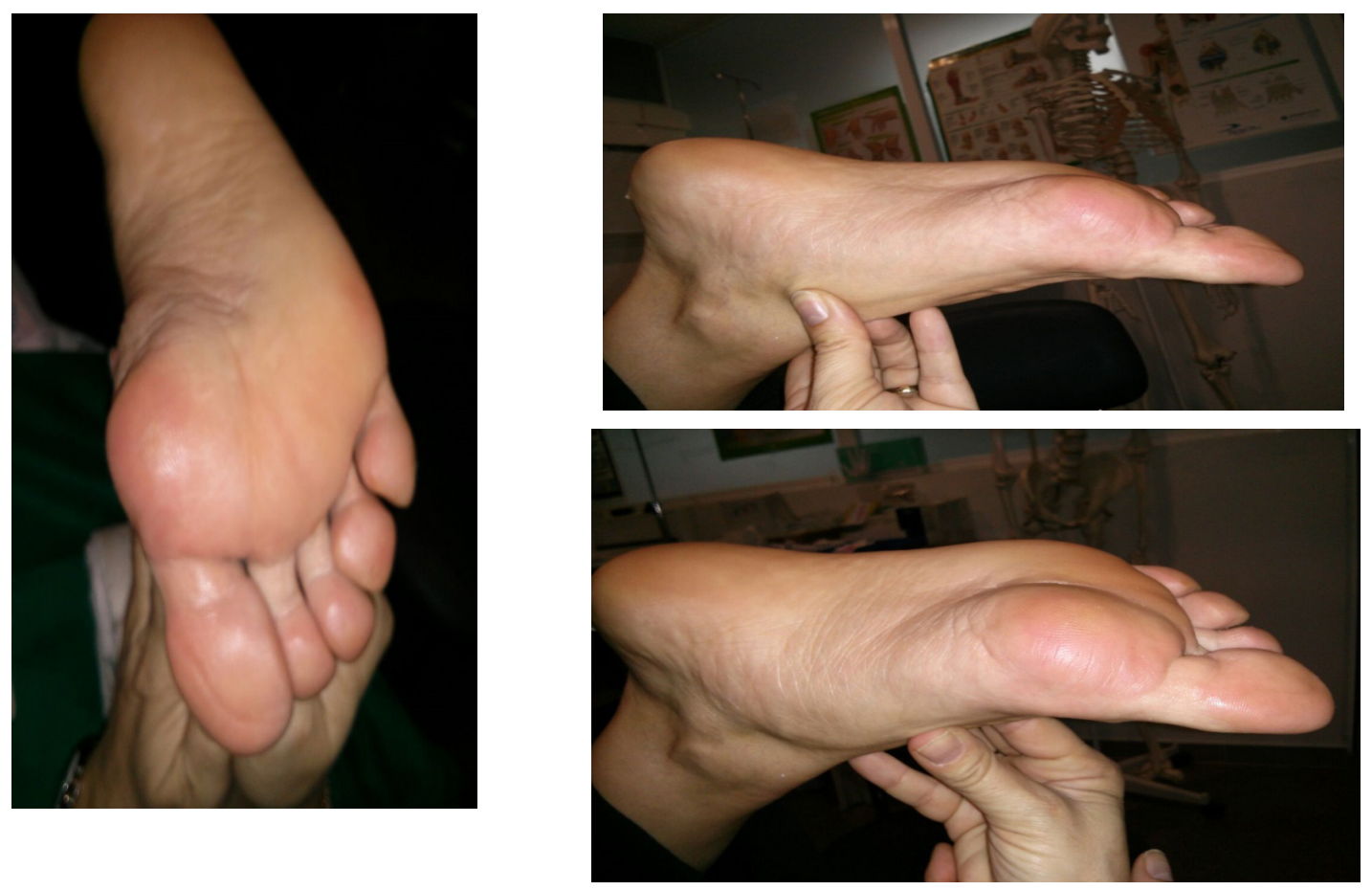

Figura 1: En la exploración se observa un importante aumento de volumen plantar a nivel de la $1^{\circ}$ AMTF, con signos de inflamación: calor, rubor, eritema... Refiere dolor con la palpación directa así como a la movilidad de la articulación, la cual no está disminuida; flexión dorsal de $60^{\circ}$ y flexión plantar de $45^{\circ}$.

En las pruebas complementarias que aporta de Urgencias se observa: Radiografía dorsoplantar muestra una valoración goniométrica alterada, para el ángulo de Meschan, con sesamoideo medial bipartito, con bordes irregulares como se aprecia en la (Figura .2).

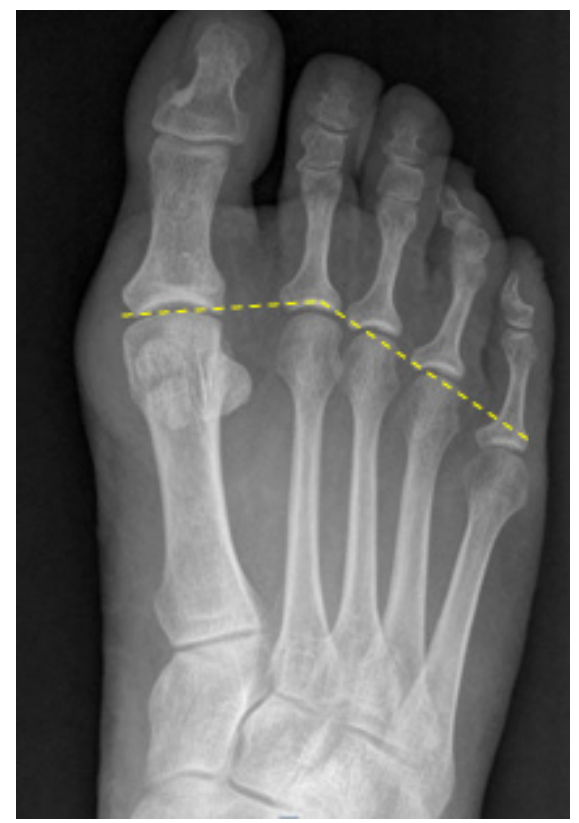

En la Proyección lateral (Figura 3), se observa un pie cavo idiopático, con escafoides accesorio tipo 2. Y en los sesamoideos signos compatibles con una enfermedad de Renander.

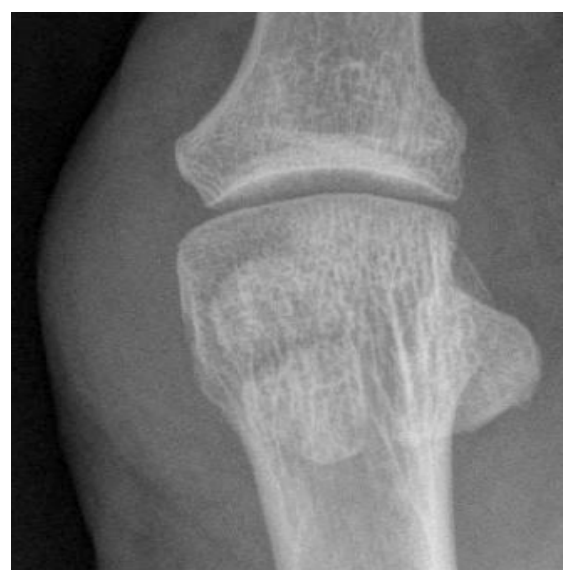


Figura 2: La radiografía dorsoplantar muestra una valoración goniométrica alterada para el ángulo de Meschan, que se encuentra aumentado, sesamoideo medial bipartito con bordes irregulares y aumento en partes blandas.
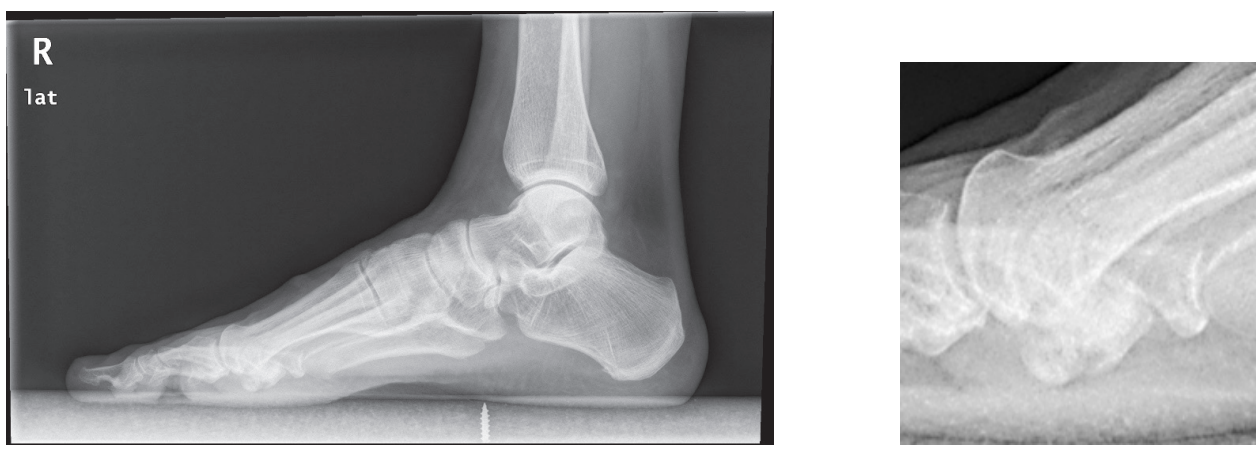

Figura 3. Proyección lateral en carga muestra un pie con dorso giboso compatible con un pie cavo idopático, presenta escafoides accesorio tipo 2. En los sesamoideos se aprecian zonas líticas y esclerosis del fragmento proximal ( Renander ) y el aumento de partes blandas.

En la consulta de podología se le realiza una ecografía con diferentes cortes y eco-Doppler (figura 4,5,6,7) cuya valoración hace posible el diagnostico ecográficamente de bursitis adventicia, patología del sesamoideo, con sesamoideo medial bipartito y afectación con
Renander., en estrecha relación con la bursa, en la que el eco-doppler,muestra intensidad de grado tres, tanto perilesional, como en su interior, lo que nos indica una gran actividad inflamatoria de la lesión.

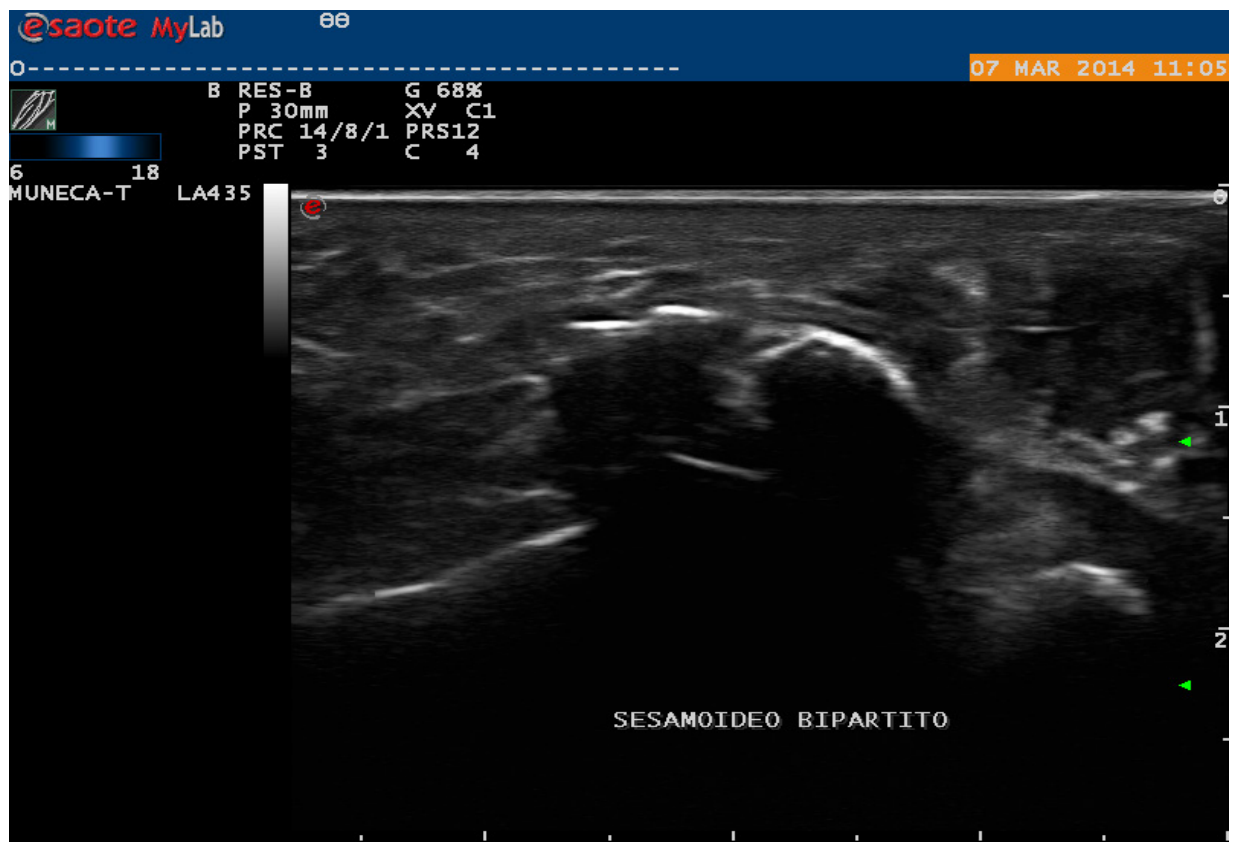

Figura 4. Corte ecográfico transversal plantar, sobre sesamoideos, donde se observa el tendón flexor del primer dedo y sobre él una masa aplanada hipoecogénica, compresible, bien delimitada, que interrumpe focalmente el plano graso subcutáneo, que se extiende en cortes más distales. 


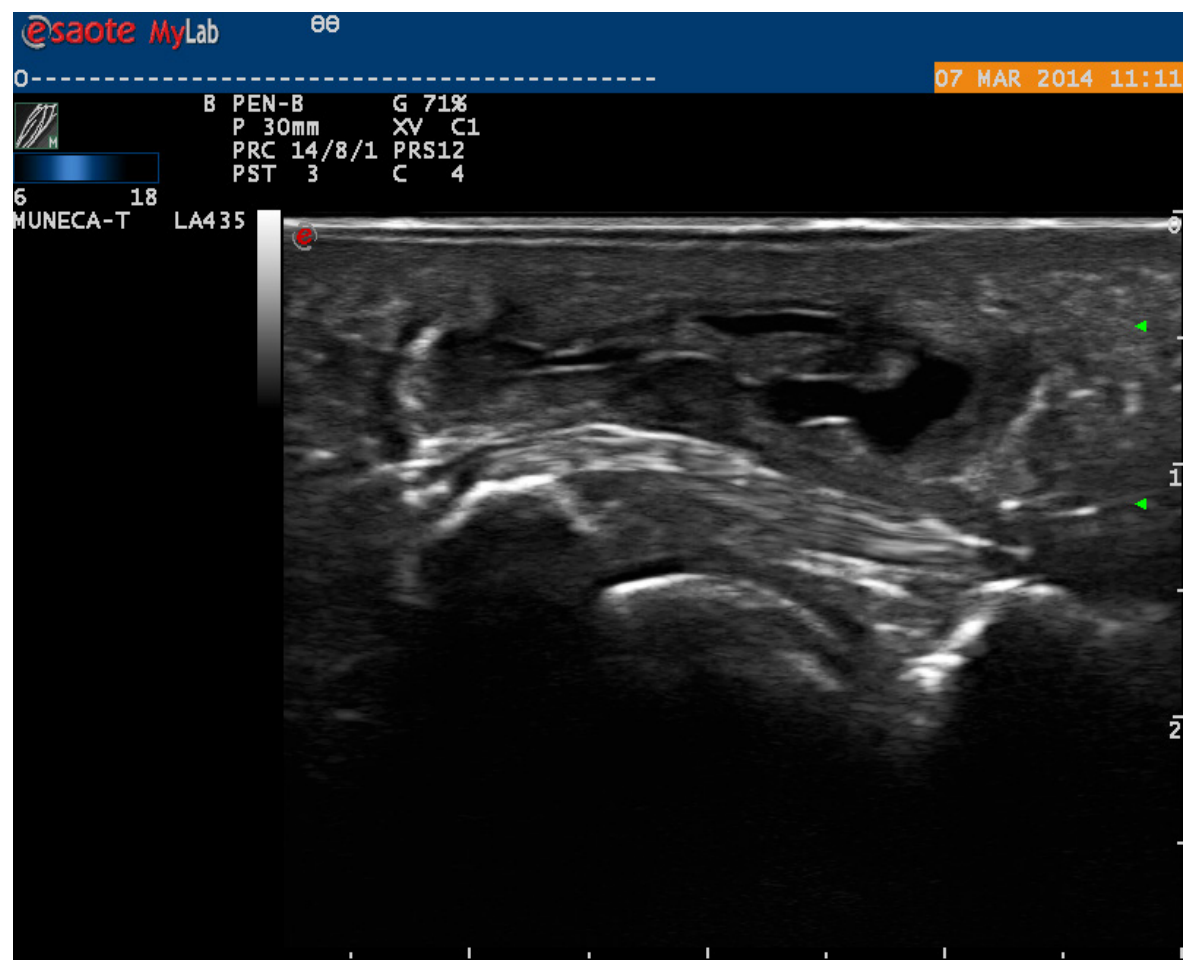

Figura 5.- El corte longitudinal plantar sobre el sesamoideo de la 1 aarticulación MTF muestra un sesamideo fragmentado y en estrecha relación con él una masa aplanada con ecotextura mixta (liquido e hipertrofia sinovial) localizada en la grasa parda de la planta del pie, de mismas características que en los otros cortes.

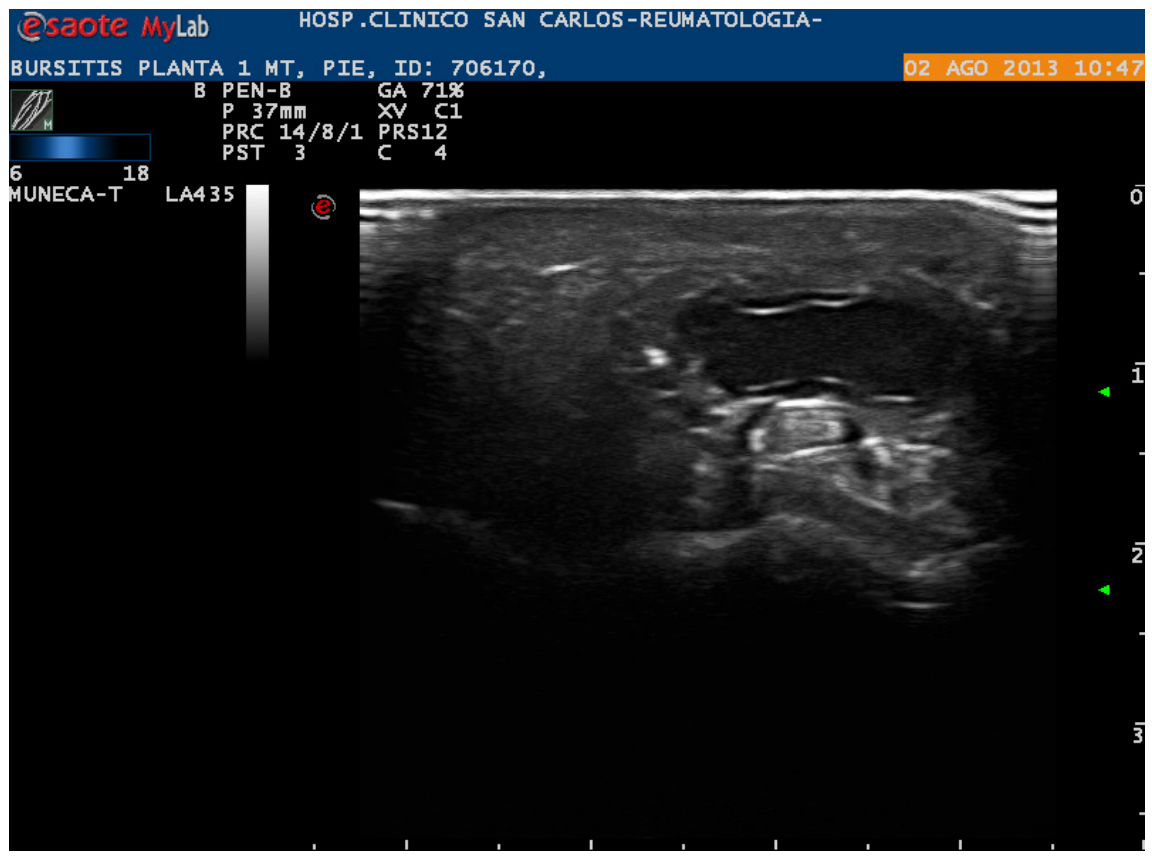

Figura 6.- Corte longitudinal plantar dinámico, presenta las mismos hallazgos, se observa la articulación MTF1 con los sesamoideos y la bursa que se extiende sobre toda la superficie. 


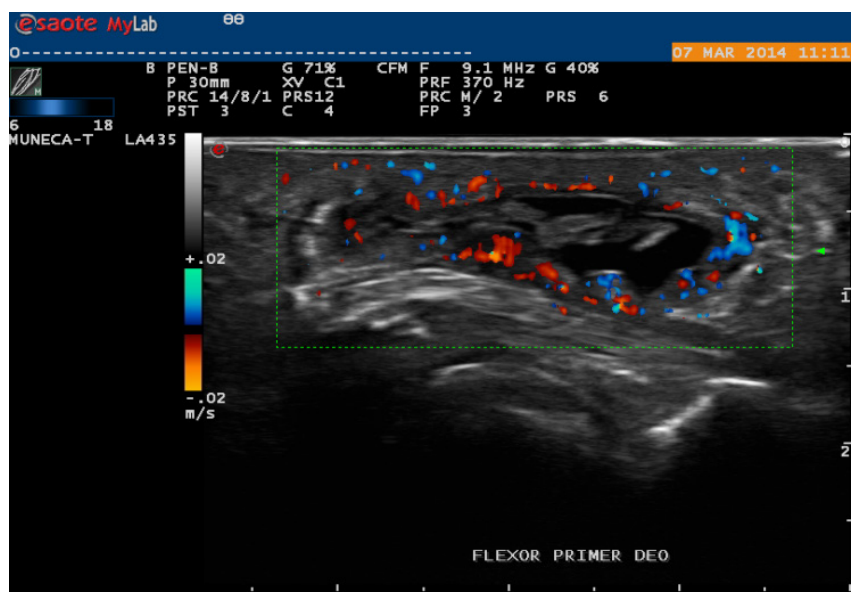

Figura 7. El estudio con eco-doppler,muestra intensidad de grado tres, tanto perilesional como en su interior, lo que nos indica la gran actividad inflamatoria de la lesión.

El tratamiento consiste en una descarga primaria con fieltros adhesivos de $1,8 \mathrm{~mm}$, en forma de media luna retro-sesamoideo, que la paciente tolera perfectamente refiriendo ausencia de dolor al caminar. (Figura 8)

Como tratamiento secundario, a los 15 días se aplica una ortesis plantar que presenta como principal elemento, la descarga de M1 retrosesamoideo y, calzado en cuña con ligero balancín, se le prohíbe usas zapatillas de andar por casa.

El seguimiento de la paciente a los seis meses es favorable, con ausencia de dolor, e inflamación. Escala de $\mathrm{EVA}^{0}$.
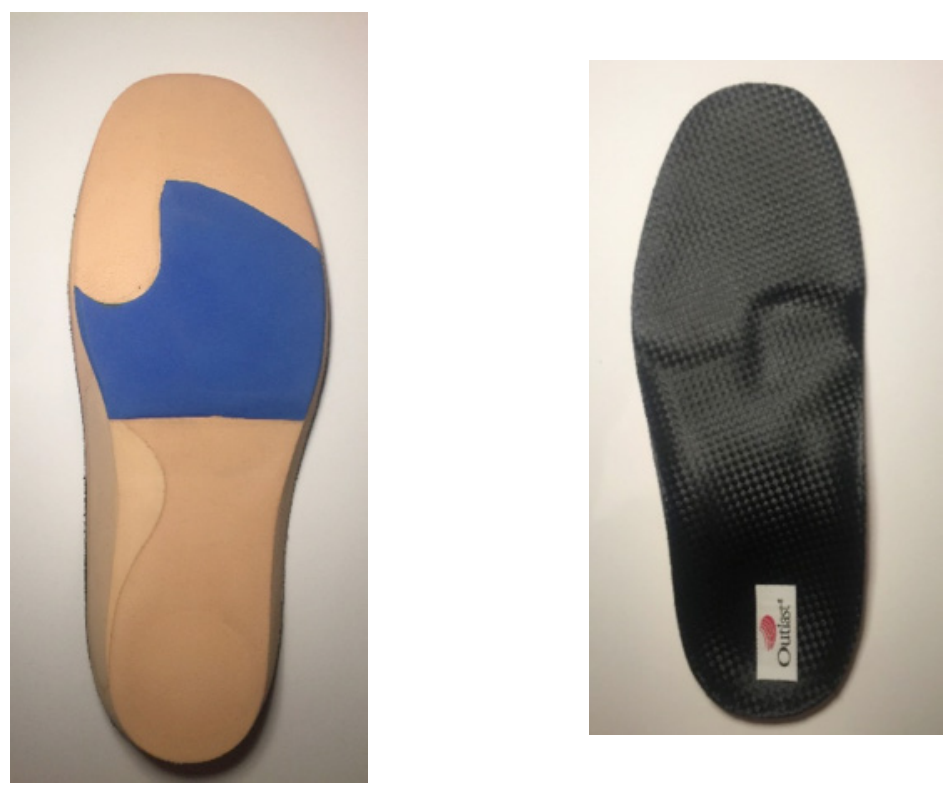

Figura 8.-.Ortesis plantar con descarga especifica de M1 y sesamoideos. 


\section{Discusión}

En el caso clínico aportado la localización de la bursitis adventicia es a nivel de los sesamoideos, localización descrita por varios autores ${ }^{1,2}$.

En nuestra paciente la posible etiología es la presencia de una morfología estructural de pie cavo, asociado a la plantar flexión anormal del primer radio, etiología descrita por numerosos autores $^{5-7}$.

Entendemos que la RM está considerada como prueba de elección en examen diagnóstico ${ }^{10}$, donde las bursas inflamadas se muestran como bolsas bordeadas por una capa de tejido de espesor variable con realce periférico después de la administración intravenosa de un agente de contraste a base de gadolinio $^{1,3,4,11,12}, \quad o$, sin borde exterior que muestra la captación de contraste difusa sin realce periférico, por lo que, es difícil diferenciar entre bursitis abierta, adventicia o infecciosa; en estos casos, la clínica sugiere la etiología ${ }^{3}$.

Pero en nuestro caso se ha realizado el diagnostico con ultrasonido (US) pues este método diagnóstico es reconocido como una valiosa prueba en la identificación de patologías de tejidos blandos que afectan al $\mathrm{pie}^{13}$. Y puede revelar cambios inflamatorios, así como, colecciones de fluidos de tejidos blandos $^{3}$ que suelen ser heterogéneos. Por lo que, existe un alta sensibilidad y especificidad en el diagnóstico de esta patología con ecografía .

Como se describe en la literatura la bursa adventicia muestra un fluido hipoecoico o anecoico compresible, con una distensión heterogénea con líquido mixto, hipertrofia sinovial e hiperemia circundante ${ }^{14}$. La herramienta de la señal Doppler va ha permitir valorar de forma objetiva y eficaz grado de actividad en procesos de neovascularización sinovial como ocurre en nuestro caso indicando la existencia de una alta actividad inflamatoria en el momento de dicha prueba.
Entendemos igual que en otros estudios ${ }^{13}$ que gracias al uso de la ecografía se ha logrado proporcionar una identificación, con bajo coste insitu, e implementar de forma temprana el tratamiento. pudiendo realizar diagnóstico diferencial entre bursitis aséptica e infección mediante punción con ecografía guiada ${ }^{3}$ que en nuestro caso no ha sido necesario.

Nuestra primera elección de tratamiento ha sido el conservador como describen $y$ apoyan diversos autores ${ }^{3,6,7,15}$, utilizando elementos ortésicos como soportes plantares, modificaciones en el calzado, reduciendo la carga (pérdida de peso del paciente si fuese necesario), así como, períodos de reposo y descanso tisular acompañando ambos tratamientos junto a la administración oral de antiinflamatorios no esteroideos ${ }^{16}$.

En nuestra paciente se le realizó una descarga primaria con fieltros adhesivos durante 15 dias consiguiendo reducir no solo en contenido de la bursa, sino también, cesando la actividad inflamatoria.

A los 15 dias se aplica una ortesis plantar, que como principal elemento presenta descarga de M1 retro- sesamoideo y calzado en cuña con ligero balancín. Los resultados de nuestra paciente son excelentes sin necesidad de antinflamatorios ni aplicación de infiltración local intrarticular de cortisona con anestésico que está descrito para eliminar la sintomatología en casos recalcitrantes ${ }^{15,16} \mathrm{o}$, la sesamoidectomía en caso que el dolor no cese $^{6,16}$.

\section{Conclusión}

La ecografía musculoesqueletica es una herramienta diagnóstica en la identificación de patologías de tejidos blandos que afectan al pie con un alta sensibilidad y especificidad, bajo coste y prueba insitu en la consulta de podología, que nos permite implementar de manera rápida y eficaz un tratamiento adecuado.

\section{Bibliografía}

1. Van Hul E, Vanhoenacker F, Van Dyck P, De Schepper A, Parizel PM. Pseudotumoural soft tissue lesions of the foot and ankle: a pictorial review. Insights into Imaging. 2011; 2(4):439-452.

2. Jensen B, Leykum B, Fiorito J, Woodruff D, Bharara M, Armstrong DG. Adventitious Bursae Underlying Chronic Wounds: Another Possible Deterrent to Healing.Eplasty. 2012; 12:e14. 
3. Foisneau-Lottin A., Martinet N., Henrot P., Paysant J., Blum A., Andre J.-M Bursitis, adventitious bursa, localized soft-tissue inflammation, and bone marrow edema in tibial stumps: The contribution of magnetic resonance imaging to the diagnosis and management of mechanical stress complications Archives of Physical. Medicine and Rehabilitation, 2003. 84 (5), 770-777.

4. Studler U 1, Mengiardi B, Bode B, Schöttle PB, Pfirrmann CW, Hodler J, Zanetti M. Fibrosis and Adventitious Bursae in Plantar Fat Pad of Forefoot: MR Imaging Findings in Asymptomatic Volunteers and MR Imaging-Histologic Comparison. Radiology. 2008 ; 246 (3): 863 - 70.

5. Nwawka OK, Hayashi D, Diaz LE, et al. Sesamoids and accessory ossicles of the foot: anatomical variability and related pathology. Insights into Imaging. 2013;4(5):581-593.

6. Richardson EG.; Hallucal sesamoid pain: causes and surgical treatment. J Am Acad Orthop Surg. 1999 7(4):270-8.

7. Burns, J, Landorf, KB, Ryan, MM, Crosbie, J, Ouvrier, RA. Interventions for the prevention and treatment of pes cavus. Cochrane Database Syst Rev. 2007.

8. Jensen MP1, McFarland CA, Increasing the reliability and validity of pain intensity measurement in chronic pain patients. Pain. 1993; 55(2):195-203.

9. Campos PP. Diagnóstico de imagen. En: Núñez-Samper M, Llanos ALF. Biomecánica, medicina y cirugía del pie. Barcelona: Masson, 1997: 108-115

10. Ammitzbøll-Danielsen M, Janta I, Torp-Pedersen S, Naredo E, Østergaard M, Terslev L. Threedimensional Doppler ultrasound findings in healthy wrist and finger tendon sheaths - can feeding vessels lead to misinterpretation in Doppler-detected tenosynovitis? Arthritis Research \& Therapy. 2016; 18:70.

11. Kier R., Abrahamian H., Caminear D., Eterno R., Feldman A., Abrahamsen T, Davis D., Rice American MR Arthrography of the Second and Third Metatarsophalangeal Joints for the Detection of Tears of the Plantar Plate and Joint Capsule.American Journal of Roentgenology 2010 194:4, 1079-1081

12. Suárez C., Fraga G. Patología más frecuente causante de dolor en el antepie diagnosticada por Resonancia Magnética. Revista Síntesis 2014. Vol I - No 3

13. Jones S., Phillips M. Early Identification of Foot and Lower Limb Stress Fractures using Diagnostic Ultrasonography: A review of 3 cases. The Foot and Ankle Online Journal 2010.3 (4): 3

14. Ruangchaijatuporn T., Gaetke-Udager K., Jacobson J., Yablon C., Morag Y. Ultrasound evaluation of bursae: anatomy and pathological appearances. Skeletal Radiology 2017.;46 (4):445-462.

15. Kumar S, Kadavigere R, Puppala R, Ayachit A, Singh R. Subhallucal Interphalangeal Sesamoiditis: A Rare Cause of Chronic Great Toe Pain. Journal of Clinical and Diagnostic Research: JCDR. 2015;9(5):TD01-TD02.

16. Carranza, A. Lesiones traumáticas de los sesamoideos de la articulación metatarsofalángica. Avances Traum. 1998; 28: 234-236 